\title{
Calculation of point-defect entropy in metals
}

\author{
YuRi Mishin† \\ School of Computational Sciences, MSN 5C3, George Mason University, \\ Fairfax, Virginia 22030, USA \\ Mads R. Sørensen $\ddagger$ and Arthur F. Voter \\ Theoretical Division, Los Alamos National Laboratory, Los Alamos, \\ New Mexico 87545, USA
}

[Received 27 June 2000 and accepted in revised form 19 January 2001]

\begin{abstract}
We investigate previously used methods and propose a new method for atomistic calculations of point-defect entropies in metals within the harmonic approximation to lattice vibrations. The key problem is to predict accurately the defect formation entropy in a macroscopic crystal from atomistic calculations performed on a small system containing relatively few atoms. The results of atomistic calculations may depend significantly on the system size, geometry and boundary conditions. Two previously used methods, which we call the supercell and embedded-cluster methods, are analysed in two ways: firstly, within a linear elasticity model of a point defect and, secondly, by atomistic calculations for a vacancy and an interstitial in copper using an embedded-atom potential. The results of atomistic calculations confirm the linear elasticity analysis and show that the supercell method is much more accurate than the embedded-cluster method. However, the latter is useful for computing the defect core entropy, which turns out to be a well-defined physical quantity. We propose a new method of defect entropy calculations that combines the embedded-cluster method with a quasicontinuum approximation outside the cluster. This method, which we call an elastically corrected embedded-cluster method, has an accuracy comparable with that of the supercell method and extends defect entropy calculations towards larger system sizes.
\end{abstract}

\section{§1. INTRODUCTION}

Point defects mediate atomic diffusion, creep and other processes in solids at elevated temperatures (Flynn 1972). If the external pressure is zero $(p=0)$, the equilibrium atomic concentration of point defects of a certain type is given by

$$
C=\exp \left(-\frac{E-T S}{k_{\mathrm{B}} T}\right),
$$

$\dagger$ E-mail: ymishin@gmu.edu

$\ddagger$ Present address: Novo Nordisk A/S, Scientific Computing B2.1.31, Novo Nordisk Park, DK-2670 Maaloev, Denmark. 
where $T$ is the temperature, $E$ and $S$ are the defect formation energy and entropy respectively and $k_{\mathrm{B}}$ is the Boltzmann constant. Given a suitable model of atomic interactions, $E$ and $S$ can be determined by atomistic calculations in the zero-temperature limit using molecular statics and the harmonic approximation to atomic vibrations.

The physical quantities of interest are the defect energy and entropy in a macroscopic crystal under constant-pressure (usually zero-pressure) conditions. However, atomistic calculations of these quantities are limited to relatively small systems with approximately $10^{3}$ atoms because the diagonalization of large dynamic matrices is computationally demanding and associated with numerical problems. For systems of this size, results of atomistic calculations depend on the system size, geometry and boundary conditions. How should such calculations be performed in order to obtain accurate estimates of the defect properties in a macroscopic crystal? This question is the main topic of the present paper.

Different problems associated with defect entropy calculations have been reviewed by Catlow (1997), Foiles (1994) and Harding (1990). Most of the previous work was done on ionic solids, in which the electric charges of defects have a significant effect on entropy calculations. In this paper, we shall be concerned with metallic systems, in which point defects bear no electric charge. This will make our analysis less complex and will allow us to correlate our atomistic simulation results with simple calculations based on linear elasticity theory. Previous atomistic calculations of vacancy formation entropies in metals were undertaken by several workers (for example Hatcher et al. (1979), Fernandez and Monti (1993), Debiaggi et al. (1996), Fernandez et al. (1996, 2000) and Frank et al. (1996)). There are two different approaches to such calculations, which will be referred to in this paper as the 'supercell method' and the 'embedded-cluster method'. In the supercell method, all free atoms (i.e. atoms allowed to move during the energy minimization) are at the same time dynamic atoms (i.e. their vibrations are taken into account in the defect entropy calculations). $\dagger$ In the embedded-cluster method, only some of the free atoms, namely those forming a cluster centred a the defect core, are considered as dynamic, while all other free atoms are treated as static.

In this paper we investigate the supercell and embedded-cluster methods and propose an alternative approach to defect entropy calculations. In $\S 2$ we explain how entropy calculations have previously been conducted within the supercell and embedded-cluster methods. In $\S 3$ we undertake a simple linear-elasticity analysis of the energy and entropy associated with the elastic strain field around a point defect. We examine how these quantities depend on the overall system size, cluster size, and the boundary conditions imposed on the system. In $\S 4$ these dependences are compared with atomistic simulation results performed for vacancies and interstitials in copper. These simulations are also used for testing the accuracy of the entropy calculation methods considered in this paper. In $\S 5$ we present our new method for defect entropy calculations, a so-called elastically corrected embedded-cluster method. This method can be viewed as an extension of the embedded-cluster method in which the entropy of the lattice regions outside the cluster is included in a quasi-

$\dagger$ Often, especially in first-principles calculations, the term 'supercell' refers to a periodic block. In this paper, we use this term whenever all relaxed atoms are included in the entropy calculation, irrespective of the boundary conditions. 
continuum approximation. In $\S 6$ we summarize and outline possible applications of our analysis.

\section{§2. Methods of POINT-DefeCt CALCUlations}

\subsection{Defect formation energy}

In a typical atomistic simulation scheme, a point defect is created in the centre of a simulation block, and the total energy of the block is minimized with respect to atomic displacements subject to chosen boundary conditions. If the defect is a single vacancy, its formation energy can be estimated as

$$
\tilde{E}_{N}=E_{\mathrm{d}}(N-1)-E_{0}(N) \frac{(N-1)}{N},
$$

$E_{\mathrm{d}}(N-1)$ being the relaxed energy of the defected block containing $N-1$ atoms, and $E_{0}(N)$ the energy of the initial perfect-lattice block containing $N$ atoms. In this and all other equations, a tilde indicates quantities evaluated in a finite-size system. Equation (2) can be modified for other defects; for example, for an interstitial, $N-1$ should be replaced by $N+1$. If $N$ is large enough, equation (2) provides an accurate estimate of the true energy $E$ that would be found in an infinitely large system at $p=0$. Even if the block size is not very large, $E$ can be estimated by computing $\tilde{E}_{N}$ for several different $N$ values and making a linear extrapolation of the plot $\tilde{E}_{N}$ versus $1 / N$ to $1 / N \rightarrow 0$. Indeed, in metals the point-defect energy can be divided into the defect core energy $E_{\mathrm{c}}$ and the energy $E_{\mathrm{el}}$ of the elastic strain of the surrounding lattice induced by the defect:

$$
E=E_{\mathrm{c}}+E_{\mathrm{el}}
$$

The strain-energy density decreases with increasing distance $r$ from the defect as $1 / r^{6}$ (for example Hatcher et al. (1979)) (see also $\S 3$ of this paper). Thus, the total strain energy stored within a sphere of radius $R$ converges to $E_{\mathrm{el}}$ with $R \rightarrow \infty$ as $1 / R^{3}$, or as $1 / N$ assuming that $N$ is proportional to $R^{3}$. As will be shown below, this extrapolation converges to the exact point-defect energy $E$ regardless of whether the volume of the block remains fixed or is allowed to relax during the energy minimization to mimic a zero external pressure.

\subsection{Defect entropy in the supercell method}

While the point-defect energy can be calculated almost routinely, the vibrational entropy calculation is a more delicate matter. We shall first consider the supercell method of entropy calculations. This method uses a cubic or rectangular lattice block, usually (but not always) with periodic boundary conditions in all directions. The defect formation entropy is determined from the total entropy change of the block. Thus, for a vacancy in a periodic block we have

$$
\tilde{S}_{N}=S_{\mathrm{d}}(N-1)-S_{0}(N) \frac{(N-2)}{(N-1)},
$$

where $S_{\mathrm{d}}(N-1)$ is the entropy of the $(N-1)$-atom defected block after its static relaxation, and $S_{0}(N)$ is the entropy of the initial $N$-atom perfect-lattice block. In the harmonic approximation, the vibrational entropy of an $N$-atom periodic block is given by 


$$
S=-k_{\mathrm{B}} \sum_{n=1}^{3 N-3} \ln \left(\frac{h \nu_{n}}{k_{\mathrm{B}} T}\right)+(3 N-3) k_{\mathrm{B}},
$$

$\nu_{n}$ being frequencies of normal vibrations and $h$ the Planck constant. Equations (4) and (5) take into account that three eigenvalues of the dynamic matrix, out of $3 \mathrm{~N}$, are zero owing to the translational invariance of the total energy. By combining equations (4) and (5) we have

$$
\tilde{S}_{N}=-k_{\mathrm{B}} \ln \left[\prod_{n=1}^{3 N-6} \nu_{n} /\left(\prod_{n=1}^{3 N-3} \nu_{n}^{0}\right)^{(N-2) /(N-1)}\right],
$$

$\nu_{n}^{0}$ being normal vibrations of the perfect-lattice block. In calculations with fixed boundary conditions, all eigenvalues of the dynamic matrix are positive, so the righthand sides of equations (5) and (6) should be modified by increasing $N$ by 1 . For interstitials or other defects these equations must also be modified accordingly. Note that these equations are only valid at temperatures above the Debye temperature. We thus assume that the harmonic approximation remains valid at such temperatures.

In the so-called full harmonic approximation (FHA), the normal vibrational frequencies are calculated exactly by diagonalizing the dynamic matrix of the system:

$$
D_{i \alpha j \gamma}=\frac{1}{\left(m_{\alpha} m_{\gamma}\right)^{1 / 2}} \frac{\partial^{2} E}{\partial R_{i \alpha} \partial R_{j \gamma}},
$$

where the vectors $R_{i \alpha}$ and $R_{j \gamma}$ determine the equilibrium positions of atoms $\alpha$ and $\gamma$, and $m_{\alpha}$ and $m_{\gamma}$ are the respective atomic masses. Then, the normal frequencies are given by $\nu_{n}=\lambda_{n}^{1 / 2}$, where the index $n$ numbers positive eigenvalues $\lambda_{n}$ of the dynamic matrix. To simplify entropy calculations, some workers (for example LeSar et al. (1989)) proposed to use the local harmonic (Einstein's) approximation (LHA), in which the atoms are treated as independently vibrating oscillators. In this approximation, the vibrational frequencies are calculated by diagonalizing the local dynamic $3 \times 3$ matrices $D_{i \alpha j \alpha}$. There are even further simplifications of the LHA in which only diagonal elements $D_{i \alpha i \alpha}$ are taken into account, for example the second-moment approximation of the vibrational spectrum (Sutton 1989, 1992, Foiles 1994).

As in energy calculations, the finite-size effects can be eliminated by calculating $\tilde{S}_{N}$ for several different block sizes and making a linear extrapolation of $\tilde{S}_{N}$ versus $1 / N$ to $1 / N \rightarrow 0$. The convergence of this extrapolation will be shown later in this paper. It will also be shown that, similarly to the defect energy, the defect entropy can be divided into the core entropy $S_{\mathrm{c}}$ and the entropy $S_{\mathrm{el}}$ associated with elastic distortions of the surrounding lattice:

$$
S=S_{\mathrm{c}}+S_{\mathrm{el}}
$$

In contrast with the defect energy, however, the defect entropy converges to different values, denoted as $S_{p}$ and $S_{V}$, depending on whether the volume $V$ of the block is allowed to vary during the energy minimization (zero-pressure condition, $p=0$ ) or remains fixed (constant-volume condition, $V=$ constant). For practical purposes we are more interested in the zero-pressure condition. The difference between $S_{p}$ and $S_{V}$ 
originates mainly from the elastic term $S_{\mathrm{el}}$ and can be described by the relation (Flynn 1972, Hatcher et al. 1979, Catlow et al. 1981)

$$
S_{p}-S_{V}=\beta B V_{\text {rel }},
$$

$\beta$ being the thermal expansion factor at $p=0$ defined as $V^{-1}(\partial V / \partial T)_{p=0}, B$ the isothermal bulk modulus and $V_{\text {rel }}$ the relaxation volume of the defect. The latter can be determined directly as the volume change of the simulation block due to the relaxation, although other methods are also available (Finnis and Sachdev 1976, Gillan 1981). In practice, supercell calculations of defect entropy are limited to relatively small blocks $\left(N \approx 10^{3}\right)$ because the diagonalization of larger dynamic matrices becomes computationally demanding and is associated with numerical problems. This prevents the method from being applied to complex defects, defectdefect interactions and other situations in which a large size of the simulation block is essential.

\subsection{Defect entropy in the embedded-cluster method}

The embedded-cluster method takes a different approach, namely the block size is chosen as large as appropriate for the inclusion of the distorted lattice regions around the defect(s) under study, typically with $N \approx 10^{3}-10^{5}$. As before, the equilibrium configuration of the block is obtained by its static relaxation. For entropy calculations, however, a cluster of atoms enclosed by a sphere centred at a chosen defect is selected. The atoms included in the cluster are considered as dynamic, while all other atoms are considered as static. The defect formation entropy is then calculated by applying the previous equations to the dynamic cluster. For example, for the vacancy formation entropy, equation (6) takes the form

$$
\tilde{S}_{N_{*}}=-k_{\mathrm{B}} \ln \left[\prod_{n=1}^{3 N_{*}-3} \nu_{n} /\left(\prod_{n=1}^{3 N_{*}} \nu_{n}^{0}\right)^{\left(N_{*}-1\right) /\left(N_{*}\right)}\right],
$$

where $\nu_{n}^{0}$ and $\nu_{n}$ are normal vibrational frequencies of the cluster before and after the introduction of the vacancy and $N *$ is the initial number of atoms in the cluster. For an interstitial defect, $N_{*}-1$ in equation (10) should be replaced by $N_{*}+1$, while $3 N_{*}-3$ should be replaced by $3 N_{*}+3$. The cluster size must be much smaller than the overall block size and must be suitable for normal frequency calculations. The obtained entropy will obviously depend on the cluster size, which gives rise to two issues: what extrapolation must be applied for obtaining a size-independent entropy, and what is the physical meaning of that entropy? The scheme previously proposed (Hatcher et al. 1979) and used for vacancy calculations in metals (Fernandez and Monti 1993, Debiaggi et al. 1996, Fernandez et al. 1996) suggests that the plot $\tilde{S}_{N_{*}}$ versus $1 / N_{*}$ must be linearly extrapolated to $1 / N_{*} \rightarrow 0$, and that the obtained entropy has the meaning of the defect formation entropy under constant-volume conditions $\left(S_{V}\right)$ provided that the simulation block was relaxed under a fixed volume. Our analysis described below does not confirm this scheme. Firstly, even if the total volume of the block remains fixed, the volume of the cluster generally changes during the relaxation. Moreover, this volume change is comparable with the defect relaxation volume $V_{\text {rel }}$. Secondly, both the linear-elasticity analysis $(\S 3)$ and atomistic simulations performed by Fernandez et al. (2000) and in this paper show that $\tilde{S}_{N_{*}}$ does not converge with increasing cluster size, at least not in metals. More specifically, it will be shown in this paper that the elastic part of the entropy is 
approximately proportional to $N_{*} / N, N$ being the total number of atoms in the block. Thus, the entropy diverges if formally extrapolated to $1 / N_{*} \rightarrow 0$, and converges to the defect core entropy in the $N_{*} \rightarrow 0$ limit. A more adequate procedure of entropy calculations within the cluster approach should therefore be developed.

\section{§3. LINEAR-ELASTICITY ANALYSIS}

\subsection{Elastic model of a point defect}

For the evaluation of the elastic energy and entropy associated with a point defect, the latter will be represented as a dilatation centre located in the centre of a large sphere of radius $R$ (figure 1). This sphere serves as an analogue of a simulation block used in atomistic simulations. A small spherical region of radius $r_{\mathrm{c}}$ around the defect will represent the defect core, while the region outside the core, $r>r_{\mathrm{c}}$, will represent the elastically strained lattice around the defect. For simplicity, the lattice will be treated as an isotropic elastic medium with Young's modulus $E$ and Poisson's ratio $\nu$. The volume effect associated with the defect formation will be modelled by a small expansion or contraction of the core, resulting in a change in its radius $r_{\mathrm{c}}$ by a small amount $u_{\mathrm{c}}$. The elastic strain field induced by this dilatation is described by the displacement function (Eshelby 1956, Landau and Lifshitz 1986)

$$
u(r)=a r+\frac{b}{r^{2}},
$$

with $u\left(r_{\mathrm{c}}\right)=u_{\mathrm{c}}$. The coefficients $a$ and $b$ depend on $u_{\mathrm{c}}$ and the boundary condition at the surface of the sphere. The first term in equation (11) represents the finitesize effect and vanishes when $R \rightarrow \infty$. Expressions for $a$ and $b$ under $p=0$ and $V=$ constant conditions at $r=R$ are given in Appendix A.

From equation (11), the principal components $\varepsilon_{r r}, \varepsilon_{\theta \theta}$ and $\varepsilon_{\phi \phi}(\theta$ and $\phi$ being polar angles) of the strain tensor are

$$
\varepsilon_{r r}=a-\frac{2 b}{r^{3}}, \quad \varepsilon_{\theta \theta}=a+\frac{b}{r^{3}},
$$

and $\varepsilon_{\phi \phi}=\varepsilon_{\theta \theta}$, while the local dilatation equals

$$
\Delta=\varepsilon_{r r}+2 \varepsilon_{\theta \theta}=3 a \text {. }
$$

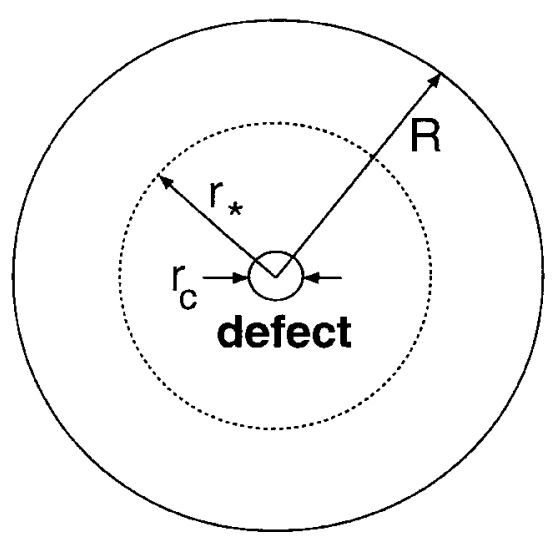

Figure 1. Schematic spherical model of a point defect for elasticity theory calculations. 
Note that this dilatation is homogeneous and arises exclusively owing to image forces.

\subsection{Relaxation volume of a point defect}

The volume change of the sphere, which represents the relaxation volume of the defect, equals

$$
\tilde{V}_{\text {rel }}(R)=4 \pi R^{2} u(R)=4 \pi\left(a R^{3}+b\right) .
$$

Under zero-pressure conditions, using expressions for $a$ and $b$ from appendix $\mathrm{A}$ and assuming that $R \gg r_{\mathrm{c}}$, we find that

$$
\tilde{V}_{\text {rel }}(R)=V_{\text {rel }}\left(1-\frac{2(1-2 \nu) r_{\mathrm{c}}^{3}}{(1+\nu) R^{3}}\right)
$$

where

$$
V_{\mathrm{rel}}=\frac{12 \pi(1-\nu) u_{\mathrm{c}} r_{\mathrm{c}}^{2}}{1+\nu}
$$

is the relaxation volume of the defect in an infinitely large system (Eshelby 1956, Flynn 1972). It is reasonable to assume that $r_{\mathrm{c}}^{3} / R^{3} \approx N_{\mathrm{c}} / N, N_{\mathrm{c}}$ being the number of atoms in the defect core. Then, equation (15) suggests that the absolute value of the relaxation volume evaluated on a finite system is slightly lower than its true absolute value, and tends to that value as $1 / N, N \rightarrow \infty$.

\subsection{Defect formation energy}

We shall now examine the defect energy. The strain energy density around the defect is given by the general expression (Landau and Lifshitz 1986)

$$
W(r)=\frac{(1-\nu) E \Delta^{2}}{2(1+\nu)(1-2 \nu)}-\frac{E\left(\varepsilon_{\theta \theta}^{2}+2 \varepsilon_{r r} \varepsilon_{\theta \theta}\right)}{1+\nu} .
$$

Using equations (12) we obtain

$$
W(r)=\frac{3 E a^{2}}{2(1-2 \nu)}+\frac{3 E b^{2}}{(1+\nu) r^{6}} .
$$

The elastic strain energy stored in the sphere equals

$$
\tilde{E}_{\mathrm{el}}(R)=4 \pi \int_{r_{\mathrm{c}}}^{R} W(r) r^{2} \mathrm{~d} r=4 \pi E\left(R^{3}-r_{\mathrm{c}}^{3}\right)\left(\frac{a^{2}}{2(1-2 \nu)}+\frac{b^{2}}{(1+\nu) r_{\mathrm{c}}^{3} R^{3}}\right) .
$$

In the limit of an infinitely large system we have $a \rightarrow 0$ and $b \rightarrow u_{\mathrm{c}} r_{\mathrm{c}}^{2}$ (see appendix A), and equation (18) gives $W(r) \propto 1 / r^{6}$ (Hatcher et al. 1979). In this limiting case, equation (19) reduces to the well-known expression (for example Eshelby (1956))

$$
E_{\mathrm{el}}=\frac{(1+\nu) E V_{\mathrm{rel}}^{2}}{27(1-\nu)^{2} V_{0}}
$$

where $V_{0}=4 \pi r_{\mathrm{c}}^{3} / 3$ is the defect core volume. In a finite system, $\tilde{E}_{\mathrm{el}}(R)$ will depend on the boundary condition at $r=R$. Using the expressions for $a$ and $b$ from appendix A and limiting our calculations to linear terms in $\left(r_{\mathrm{c}} / R\right)^{3}$, we find that 


$$
\tilde{E}_{\mathrm{el}, p}(R)=E_{\mathrm{el}}\left(1-\frac{3(1-\nu) r_{\mathrm{c}}^{3}}{(1+\nu) R^{3}}\right)
$$

for $p=0$ and

$$
\tilde{E}_{\mathrm{el}, V}(R)=E_{\mathrm{el}}\left(1+\frac{3(1-\nu) r_{\mathrm{c}}^{3}}{2(1-2 \nu) R^{3}}\right)
$$

for $V=$ constant. These equations confirm that, as the block size increases, the defect formation energy tends to the same value, $E_{\mathrm{c}}+E_{\mathrm{el}}$, regardless of the boundary condition. The asymptotic behaviour is always $1 / N$, but the slope $\mathrm{d} \tilde{E} / \mathrm{d}(1 / N)$ and even its sign are different for $p=0$ and $V=$ constant. Relaxation under a fixed volume slightly overestimates the defect energy, while relaxation under zero pressure slightly underestimates the defect energy. In other words, we always have

$$
\tilde{E}_{p} \leqslant E \leqslant \tilde{E}_{V}
$$

where $\tilde{E}_{p}$ and $\tilde{E}_{V}$ are estimates of the true defect energy $E$ obtained on the same simulation block under $p=0$ and $V=$ constant conditions respectively. This relation does not depend on the sign of $V_{\text {rel }}$ and is valid for both vacancies $\left(V_{\text {rel }}<0\right)$ and interstitials $\left(V_{\text {rel }}>0\right)$. Note that the ratio of the slopes $\mathrm{d} \tilde{E}_{p} / \mathrm{d}(1 / N)$ and $\mathrm{d} \tilde{E}_{V} / \mathrm{d}(1 / N)$ depends on the elastic properties of the lattice only:

$$
\frac{\mathrm{d} \tilde{E}_{p}}{\mathrm{~d} \tilde{E}_{V}}=-\frac{2(1-2 \nu)}{1+\nu} .
$$

This relation assumes that the defect core energy does not depend on the boundary conditions.

\subsection{Defect formation entropy}

\subsubsection{General expressions}

Turning now to the defect entropy, the general expression for the density of entropy associated with the elastic strain is (Landau and Lifshitz 1986)

$$
\mathcal{S}=\beta B \Delta .
$$

In the context of the cluster method, we are interested in the total elastic entropy of a sphere with an intermediate radius $r_{*}$ such that $r_{\mathrm{c}}<r_{*} \leqslant R$ (figure 1). For this 'cluster' entropy we have

$$
\tilde{S}_{\mathrm{el}}\left(r_{*}, R\right)=4 \pi \int_{r_{\mathrm{c}}}^{r_{*}} \mathcal{S} r^{2} \mathrm{~d} r=4 \pi \beta B a\left(r_{*}^{3}-r_{\mathrm{c}}^{3}\right),
$$

where we used equation (13) for $\Delta$. Note that the $R$ dependence enters equation (26) through the coefficient $a$. Using the expressions for $a$ (appendix A) we find in the first order in $\left(r_{\mathrm{c}} / R\right)^{3}$

$$
\tilde{S}_{\mathrm{el}, p}\left(r_{*}, R\right)=S_{\mathrm{el}, p} \frac{r_{*}^{3}-r_{\mathrm{c}}^{3}}{R^{3}}\left(1-\frac{2(1-2 \nu) r_{\mathrm{c}}^{3}}{(1+\nu) R^{3}}\right)
$$

for $p=0$ and 


$$
\tilde{S}_{\mathrm{el}, V}\left(r_{*}, R\right)=S_{\mathrm{el}, V} \frac{r_{*}^{3}-r_{\mathrm{c}}^{3}}{R^{3}}\left(1+\frac{r_{\mathrm{c}}^{3}}{R^{3}}\right)
$$

for $V=$ constant. In these equations,

$$
S_{\mathrm{el}, p}=\frac{2(1-2 \nu)}{3(1-\nu)} \beta B V_{\mathrm{rel}}
$$

and

$$
S_{\mathrm{el}, V}=-\frac{1+\nu}{3(1-\nu)} \beta B V_{\mathrm{rel}}
$$

are the asymptotic values of $\tilde{S}_{\mathrm{el}}\left(r_{*}, R\right)$ when $r_{*}=R \rightarrow \infty$. Obviously, they have the meaning of the elastic parts of defect formation entropy in an infinitely large system under $p=0$ and $V=$ constant conditions respectively. Note that they satisfy the relation

$$
S_{\mathrm{el}, p}-S_{\mathrm{el}, V}=\beta B V_{\mathrm{rel}},
$$

which is identical with equation (9) provided that the core entropy is independent of the boundary condition. Thus, our elastic model provides more specific expressions for $S_{p}$ and $S_{V}$ than equation (9), namely

$$
S_{p}=S_{\mathrm{c}}+\frac{2(1-2 \nu)}{3(1-\nu)} \beta B V_{\mathrm{rel}}
$$

and

$$
S_{V}=S_{\mathrm{c}}-\frac{1+\nu}{3(1-\nu)} \beta B V_{\text {rel }} .
$$

\subsubsection{Supercell method}

The case of $r_{*}=R \gg r_{\mathrm{c}}$ corresponds to the supercell method of atomistic simulations. In this case, when $R \rightarrow \infty$, the entropy tends to either $S_{p}$ or $S_{V}$ depending on the boundary condition. More precisely, to first order in the small parameter $\left(r_{\mathrm{c}} / R\right)^{3}$, equations (27) and (28) give

$$
\tilde{S}_{\mathrm{el}, p}(R, R)=S_{\mathrm{el}, p}\left(1-\frac{3(1-\nu) r_{\mathrm{c}}^{3}}{(1+\nu) R^{3}}\right)
$$

for $p=0$ and

$$
\tilde{S}_{\mathrm{el}, V}(R, R)=S_{\mathrm{el}, V}
$$

for $V=$ constant. In the latter case, finite-size corrections are predicted to be of the order of $\left(r_{\mathrm{c}} / R\right)^{6}$ or higher. These equations suggest that the defect entropy determined by the supercell method must converge very rapidly, that is at least as fast as the defect energy. Equation (34) also suggests that, under zero-pressure conditions, the obtained entropy can be refined by a linear extrapolation of the plot $\tilde{S}$ versus $1 / N$ to $1 / N \rightarrow 0$. In practice, this turns out to be also valid for the constant-volume condition $(\S 4.3)$. 


\subsubsection{Embedded-cluster method}

The embedded-cluster scheme of atomistic simulations corresponds to $r_{\mathrm{c}} \ll r_{*} \ll R$. In this case equations (27) and (28) become

$$
\tilde{S}_{\mathrm{el}, p}\left(r_{*}, R\right)=\left(\frac{r_{*}}{R}\right)^{3} S_{\mathrm{el}, p}
$$

and

$$
\tilde{S}_{\mathrm{el}, V}\left(r_{*}, R\right)=\left(\frac{r_{*}}{R}\right)^{3} S_{\mathrm{el}, V}
$$

respectively. These equations show that the elastic part of the entropy determined by the embedded-cluster method is approximately proportional to $N_{*} / N$. In the limit of a relatively small cluster or a relatively large supercell $\left(N_{*} / N \rightarrow 0\right)$, this method converges to the defect core entropy. In the limit of $1 / N_{*} \rightarrow 0$ (Hatcher et al. 1979) the entropy diverges, which casts doubts on the extrapolation scheme used previously (Hatcher et al. 1979, Fernandez and Monti 1993, Debiaggi et al. 1996, Fernandez et al. 1996). Instead, the true elastic entropy of the defect can be obtained as the slope

$$
\frac{\mathrm{d} \tilde{S}}{\mathrm{~d}\left(N_{*} / N\right)}
$$

Knowing also $S_{\mathrm{c}}$ from the extrapolation to $N_{*} / N \rightarrow 0$, we can determine the full entropy $S$ from equation (8). This scheme is essentially equivalent to a linear extrapolation of the plot $\tilde{S}$ versus $N_{*} / N$ to $N_{*} / N \rightarrow 1$, that is extrapolation of the embedded-cluster scheme to its supercell limit. This extrapolation has recently been discussed by Fernandez et al. (2000).

\section{§4. Атоmistic Simulations}

\subsection{Simulation procedures}

Atomistic simulations were performed for a vacancy and an interstitial in copper using an embedded-atom potential constructed by Voter $(1993,1998)$. The potential generation procedure was described elsewhere (Voter 1993, 1994). The potential is fitted to reproduce accurately experimental values of the equilibrium lattice period, cohesive energy, elastic constants, vacancy formation energy and other properties of copper. The elastic constants $c_{i j}$ and bulk modulus $B=\left(c_{11}+2 c_{12}\right) / 3$ predicted by the potential are given in table 1 .

For comparison with the isotropic elasticity calculations ( $\$ 3)$ we need to evaluate Poisson's ratio for copper. Because the elastic properties of copper are strongly

\begin{tabular}{|c|c|c|c|c|c|c|c|}
\hline \multirow{2}{*}{$\begin{array}{c}c_{11} \\
\left(\mathrm{eV} \AA^{-3}\right)\end{array}$} & \multirow{2}{*}{$\begin{array}{c}c_{12} \\
\left(\mathrm{eV} \AA^{-3}\right) \\
\end{array}$} & \multirow{2}{*}{$\begin{array}{c}c_{44} \\
\left(\mathrm{eV} \AA^{-3}\right)\end{array}$} & \multirow{2}{*}{$\begin{array}{c}B \\
\left(\mathrm{eV} \AA^{-3}\right) \\
\end{array}$} & \multirow{2}{*}{$\begin{array}{c}\mu \\
\left(\mathrm{eV}^{-3}\right)\end{array}$} & \multirow[b]{2}{*}{$\nu$} & \multicolumn{2}{|c|}{$\beta \times 10^{-5}$} \\
\hline & & & & & & LHA & FHA \\
\hline 1.1192 & 0.7690 & 0.5057 & 0.8857 & 0.3404 & 0.3466 & 5.621 & 5.479 \\
\hline
\end{tabular}

Table 1. Elastic constants and moduli and thermal expansion of copper predicted by the embedded atom potential used in this work (Voter 1993). 
anisotropic (anisotropy ratio $A=2 c_{44} /\left(c_{11}-c_{12}\right) \approx 2.89$ ), the shear modulus $\mu$ and Poisson's ratio $\nu$ significantly depend on the crystallographic direction. In particular, $\mu$ varies between $\mu=\left(c_{11}-c_{12}\right) / 2=0.1751 \mathrm{eV}^{-3}$ and $\mu=c_{44}=0.5057 \mathrm{eV}^{-3}$. We estimated $\mu$ as an average between these values, that is $\mu=0.3404 \mathrm{eV}^{-3}$. Using this value of $\mu$, we obtained $\nu$ as $\nu=c_{12} / 2\left(\mu+c_{12}\right) \approx 0.3466$.

The thermal expansion factor $\beta$ at $T \rightarrow 0$ was determined from the thermodynamic relation (Landau and Lifshitz 1980)

$$
\left(\frac{\partial S}{\partial V}\right)_{T}=\beta B .
$$

The entropy $S$ of a periodic perfect-lattice block was calculated from equation (5) under various volume expansions ranging from 0 to $0.5 \%$, and the derivative in equation (39) was evaluated by a linear approximation of the plot of $S$ versus $V$. The calculation were performed in both the FHA and the LHA approximations, in each case for various block sizes from $N=4$ to $N=864$. The $\beta$ values obtained on blocks with $N \geqslant 32$ were found to be almost identical; so the results for $N=864$ were taken as converged values and used in all further calculations (table 1).

The point defects were simulated by molecular statics in a cubic block (supercell) with periodic boundary conditions. The interstitial was considered in a split [100] dumbbell configuration, which was found to be the lowest-energy configuration. The defect formation energies were calculated as discussed in $\S 1$. The defect entropies were calculated by both the supercell and the embedded-cluster methods, in each case in both the LHA and the FHA approximations. The results are presented below.

\subsection{Point-defect energies and relaxation volumes}

The point-defect energies were calculated for blocks containing from $N=32$ to $N=5324$ atoms. The obtained energies plotted against $1 / N$ show excellent linearity for $N>200$ (figure 2). Moreover, as $1 / N \rightarrow 0$, the energies obtained under zeropressure and constant-volume conditions tend to the same value, which can be identified as the defect formation energy in an infinite crystal. This behaviour is in agreement with equations (21) and (22) derived from the elastic model. There is even a reasonable quantitative agreement between the derivatives $\mathrm{d} \tilde{E}_{p} / \mathrm{d} \tilde{E}_{V}$ determined from the plots and calculated from equation (24) (table 2). This agreement confirms that the size dependence of calculated defect formation energies in metals arises primarily from elastic interactions of the defects with the block boundaries and that this dependence can be effectively eliminated by a linear extrapolation to $1 / N \rightarrow 0$. Figure 2 also shows that the volume relaxation of the block does not guarantee significantly more accurate energies. One can save computational efforts by carrying out a set of constant-volume calculations followed by the linear extrapolation to $1 / N \rightarrow 0$.

The relaxation volumes were computed as functions of $N$ (with $N$ up to 4000) both directly and from the equation $V_{\mathrm{r}} / \Omega_{0}=-P / B$, where $P$ is the sum of hydrostatic stresses on all atoms after the relaxation at $V=$ constant, and $\Omega_{0}$ is the atomic volume. (The stresses were calculated from the virial expression at $T=0$.) For $N>200$, both calculation methods gave very similar volumes, which behaved linearly with $1 / N$ as predicted by equation (15). The relaxation volumes extrapolated to $1 / N \rightarrow 0$ are given in table 2 . 


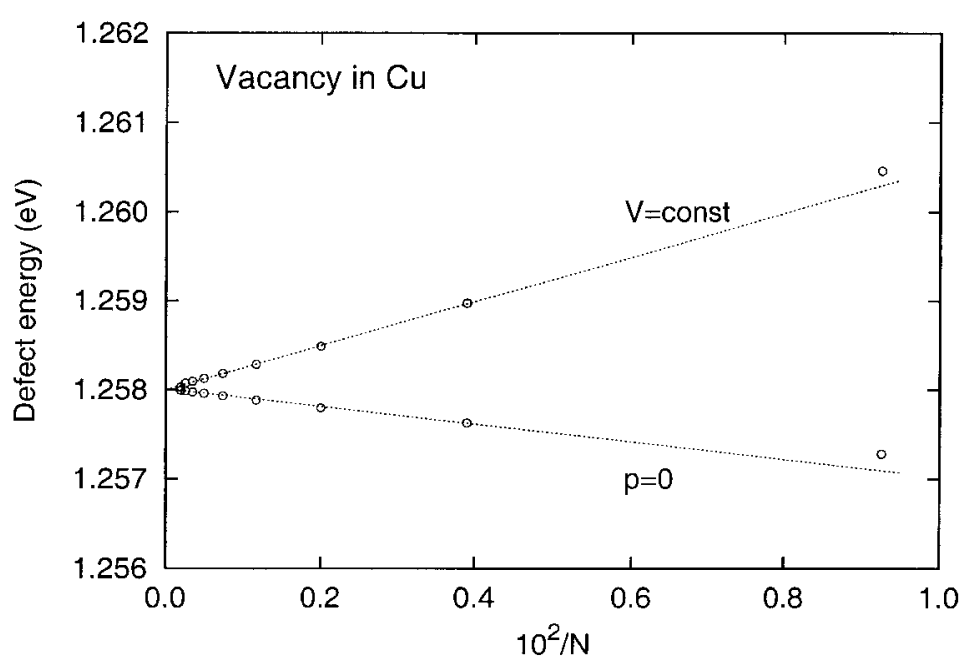

(a)

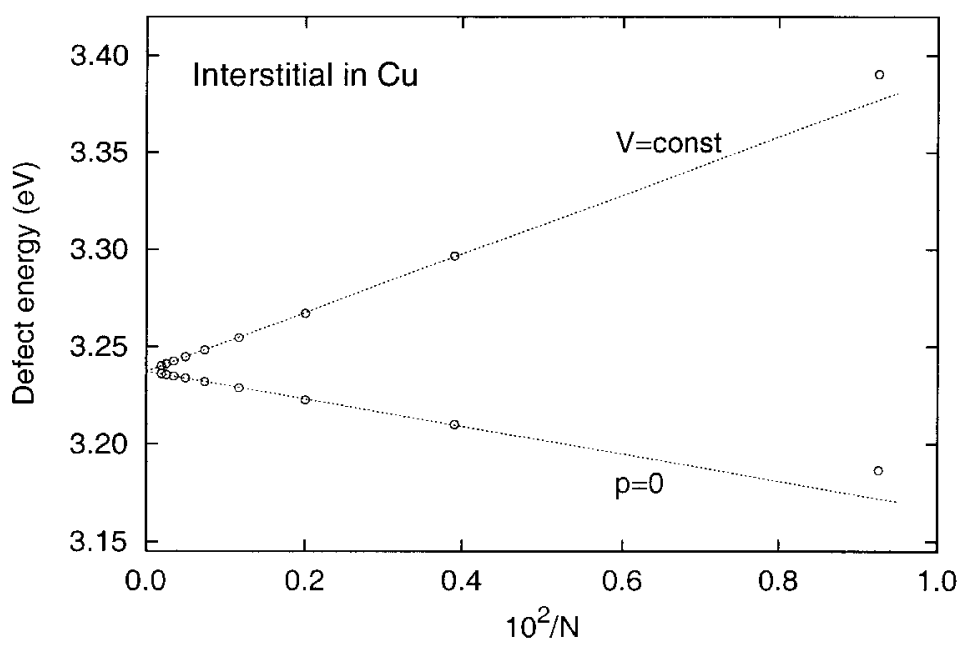

(b)

Figure 2. Calculated formation energies of $(a)$ a vacancy and $(b)$ an interstitial in copper as functions of the inverse number $1 / N$ of atoms in the simulation block. Two types of boundary condition were considered: $p=0$ and $V=$ constant. The energies relating to a macroscopic crystal are obtained by a linear extrapolation to $1 / N \rightarrow 0$.

Table 2. Calculated formation energies $E$, relaxation volumes $V_{\text {rel }}$ and derivatives $\mathrm{d} \tilde{E}_{p} / \mathrm{d} \tilde{E}_{V}$ for a vacancy and an interstitial in copper. $\Omega_{0}$ is the atomic volume.

\begin{tabular}{|c|c|c|c|c|}
\hline \multirow[b]{2}{*}{ Defect } & \multirow{2}{*}{$\begin{array}{c}E \\
(\mathrm{eV})\end{array}$} & \multirow[b]{2}{*}{$V_{\text {rel }} / \Omega_{0}$} & \multicolumn{2}{|c|}{$\mathrm{d} \tilde{E}_{p} / \mathrm{d} \tilde{E}_{V}$} \\
\hline & & & Simulation & Equation (24) \\
\hline Vacancy & 1.2580 & -0.2575 & -0.3987 & -0.4557 \\
\hline Interstitial & 3.2372 & 2.0608 & -0.4659 & -0.4557 \\
\hline
\end{tabular}




\subsection{Defect entropies from the supercell method}

Supercell calculations of defect entropies were carried out for $N=32,108,256$, 500 and 864. The LHA calculations additionally included blocks with $N=1372$ and 2048. The obtained entropies show a good linearity when plotted versus $1 / N$ for both types of boundary condition (figure 3 ). The entropies obtained by a linear extrapolation to $1 / N \rightarrow 0$ are listed in table 3 (the point for $N=32$ was not included in the fit). This table also compares the obtained differences $S_{p}-S_{V}$ with the products $\beta B V_{\text {rel }}$ appearing in equation (9). This comparison shows that equation (9) works very well for both vacancies and interstitials. Overall, the supercell method appears to be very accurate and reliable. Already a block with a few hundred atoms is sufficient for obtaining a good estimate of the defect entropy, plus this estimate can be further refined by applying the linear extrapolation scheme. Figure 3 demonstrates that the LHA provides only a poor approximation of defect entropies.

Although the observed $1 / N$ convergence of entropy is in qualitative agreement with the elasticity analysis ( $(3)$, quantitative agreement with equations (34) and (35) regarding the slopes of the lines was not observed, and in fact was not even expected in view of the approximate character of those equations. As an additional complication, in FHA calculations even the perfect-lattice entropy per atom slightly depends on the block size. In fact, similarly to the defect entropy, the perfect-lattice entropy was found to behave fairly linearly when plotted against $1 / N$. Although the reasons for this linear behaviour are different from those for the defect entropy, the variation in the perfect-lattice entropy with the block size could have affected the slopes of the plots in figure 3. Despite this discrepancy with linear-elasticity equations regarding the slopes of the lines, the supercell method provides a high accuracy and fast convergence of calculations. In the following, the defect entropies obtained by this method will be used as reference values for the evaluation of other methods.

\subsection{Defect entropies from the embedded-cluster method}

Embedded-cluster calculations of the defect entropies were performed for a large set of block and cluster sizes, typically with $N=2048$ to $N=16384$ and $N_{*}=249$ to $N_{*}=1289$. In figure 4 we plot the whole set of vacancy and interstitial entropies calculated in the FHA as functions of $N_{*} / N$. The observed linearity confirms equations (36) and (37). Because the elastic entropies $S_{\mathrm{el}, p}$ and $S_{\mathrm{el}, V}$ are proportional to $V_{\text {rel }}$ (see equations (29) and (30)), the slopes of the plots must have different signs for a vacancy and an interstitial under the same boundary condition, which is indeed observed in figure 4. Importantly, despite the scatter of the points the entropies obtained under different boundary conditions converge, as $1 / N \rightarrow 0$, to the same entropy $S_{\mathrm{c}}$ within $\pm 0.005 k_{\mathrm{B}}$. This excellent agreement demonstrates that the defect core entropy is a well-defined physical quantity that can be determined very accurately by the embedded-cluster method. Similar calculations were performed in the LHA. The linearity of the plots and convergence to the same core entropy regardless of the boundary conditions were followed with even better accuracy.

Using the slopes of the plots in figure 4, the defect entropies $S_{p}$ and $S_{V}$ were calculated by the scheme described in $\$ 3.4 .3$. Defect entropies were also calculated directly from equations (32) and (33) using the obtained $S_{\mathrm{c}}$ values. The results are listed in table 4 in comparison with defect entropies obtained by the supercell method. Note that the entropies determined from the slopes, equation (38), do follow the right trends but are not very accurate. Surprisingly, even equations (32) and (33) seem to work better despite the underlying assumptions and the uncertainty 


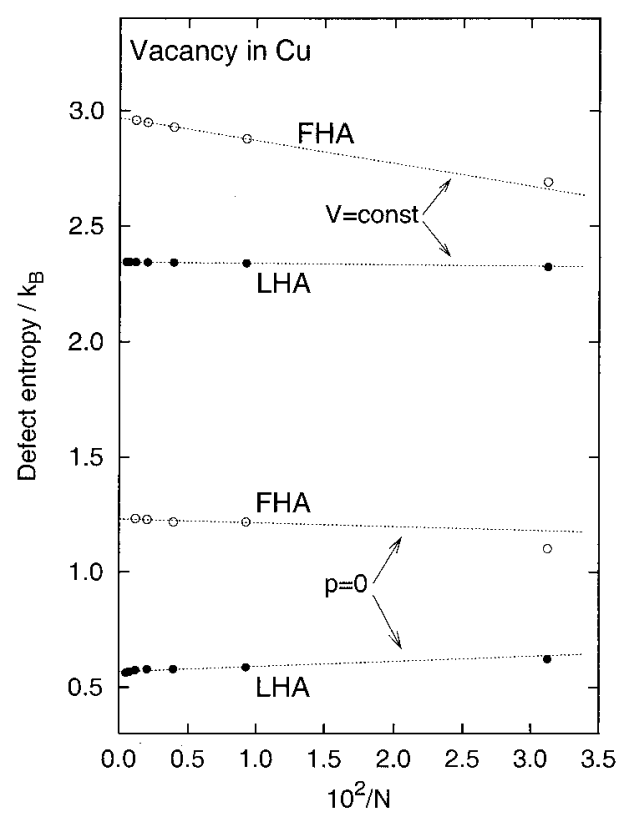

(a)

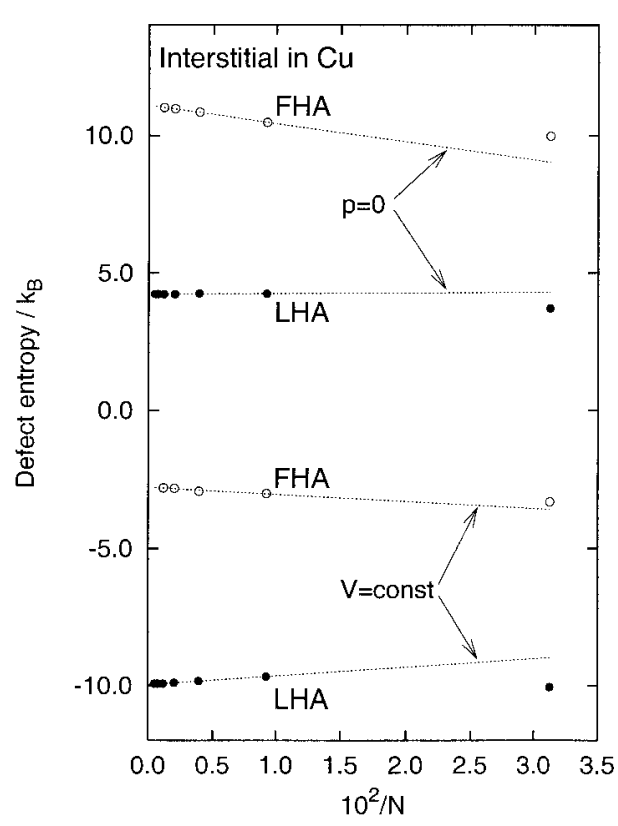

(b)

Figure 3. Calculated $(a)$ vacancy and $(b)$ interstitial formation entropies in copper as functions of the inverse number $1 / N$ of atoms in the simulation block. Calculations were performed by the supercell method in the FHA and LHA. Two types of boundary condition were considered: $p=0$ and $V=$ constant. The entropies relating to a macroscopic crystal are obtained by a linear extrapolation to $1 / N \rightarrow 0$. 
Table 3. Vacancy and interstitial formation entropies in copper calculated by the supercell method in the FHA. The differences $S_{p}-S_{V}$ and the products $\beta B V_{\text {rel }}$ are also listed for comparison (see equation (9)).

\begin{tabular}{lcccc}
\hline Defect & $\begin{array}{c}S_{p} \\
\left.\text { (units of } k_{\mathrm{B}}\right)\end{array}$ & $\begin{array}{c}S_{V} \\
\left(\text { units of } k_{\mathrm{B}}\right)\end{array}$ & $\begin{array}{c}S_{p}-S_{V} \\
\left(\text { units of } k_{\mathrm{B}}\right)\end{array}$ & $\begin{array}{c}\beta B V_{\text {rel }} \\
\text { (units of } k_{\mathrm{B}} \text { ) }\end{array}$ \\
\hline Vacancy & 1.231 & 2.969 & -1.738 & -1.713 \\
Interstitial & 11.096 & -2.791 & 13.888 & 13.707 \\
\hline
\end{tabular}

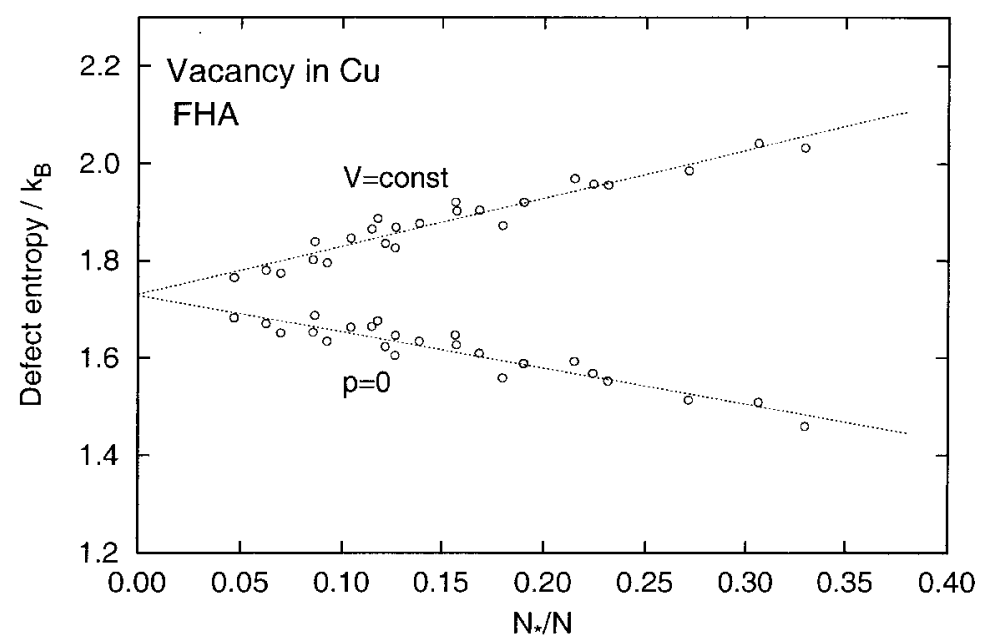

(a)

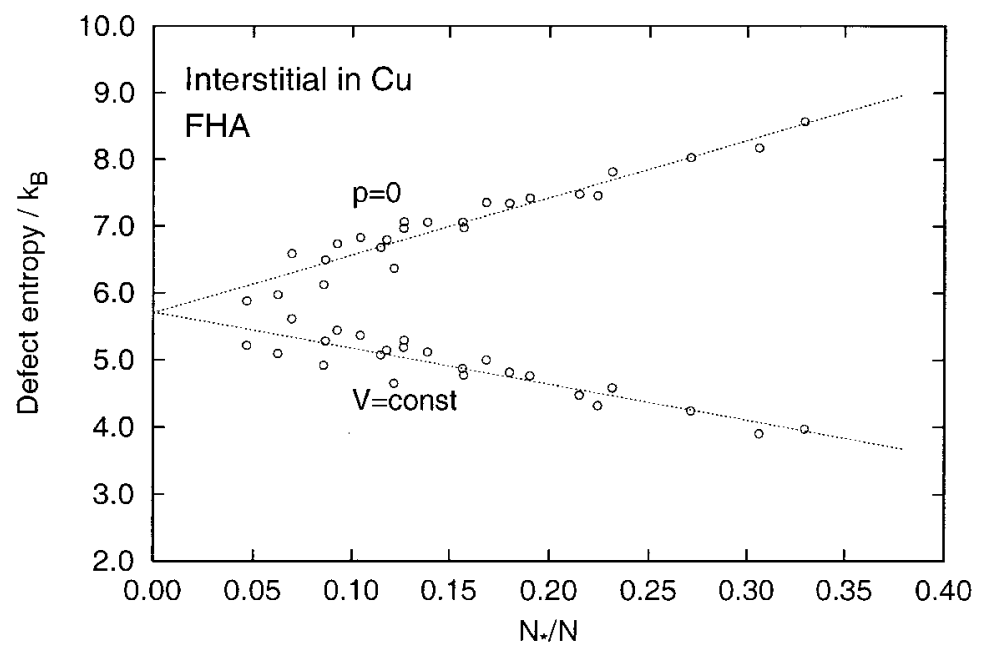

(b)

Figure 4. Formation entropies of $(a)$ a vacancy and $(b)$ an interstitial in copper calculated by the embedded-cluster method in the FHA. The entropies are plotted as functions of the ratio $N_{*} / N\left(N_{*}\right.$ is the number of atoms in the cluster, and $N$ the number of atoms in the block). Two types of boundary condition were considered: $p=0$ and $V=$ constant. In the limit $N_{*} / N \rightarrow 0$, the entropy converges to the defect core entropy regardless of the boundary condition. 
Table 4. Vacancy and interstitial formation entropies in copper calculated by the cluster method in the FHA. The entropies determined by the supercell method and calculated from equations (32) and (33) are listed for comparison.

\begin{tabular}{|c|c|c|c|c|c|c|c|}
\hline \multirow[b]{2}{*}{ Defect } & \multirow[b]{2}{*}{$\begin{array}{c}S_{\mathrm{c}} \\
\text { (units of } k_{\mathrm{B}} \text { ) }\end{array}$} & \multicolumn{3}{|c|}{$S_{p}$ (units of $\left.k_{\mathrm{B}}\right)$} & \multicolumn{3}{|c|}{$S_{\mathrm{V}}\left(\right.$ units of $\left.k_{\mathrm{B}}\right)$} \\
\hline & & Cluster & $\begin{array}{l}\text { Equation } \\
\text { (32) }\end{array}$ & Supercell & Cluster & $\begin{array}{c}\text { Equation } \\
\text { (33) }\end{array}$ & Supercell \\
\hline Vacancy & 1.729 & 0.985 & 1.193 & 1.231 & 2.718 & 2.906 & 2.969 \\
\hline Interstitial & 5.752 & 14.145 & 10.042 & 11.096 & 0.178 & -3.665 & -2.791 \\
\hline
\end{tabular}

in Poisson's ratio. These discrepancies indicate that the slopes $\mathrm{d} S / \mathrm{d}\left(N_{*} / N\right)$ are affected by factors lying beyond our elastic model, such as the cubic shape of the block and the elastic anisotropy of the lattice. Overall, the embedded-cluster method is less accurate than the supercell method even for a single defect.

\section{§5. ElasticAlly CORRECTED EMBEDDED-Cluster METHOD}

The main problem with the original embedded-cluster method is that a significant part of the defect formation entropy is stored in lattice regions outside the cluster. In order to recover that part, one has to repeat the calculations for different cluster and block sizes and apply a linear extrapolation to $N_{*} / N \rightarrow 1$. Because this extrapolation relies on the spherical symmetry and other approximations, the method is not only computationally expensive but rather inaccurate too.

We propose an alternative version of the embedded-cluster method that cures its main problem by including the outside part of the entropy in a quasicontinuum approximation. Indeed, returning for a moment to the spherical model of $\S 3$, the full entropy of defect formation equals

$$
S=S_{\mathrm{c}}+4 \pi \int_{r_{\mathrm{c}}}^{r_{*}} \mathcal{S} r^{2} \mathrm{~d} r+4 \pi \int_{r_{*}}^{R} \mathcal{S} r^{2} \mathrm{~d} r .
$$

The first two terms represent the entropy change of an embedded cluster and, in atomistic calculations, are approximated by the harmonic entropy $\tilde{S}_{N_{\star}}$ given by equation (10). The last term represents the elastic entropy associated with distorted lattice regions around the cluster. If the lattice distortions are small, the associated entropy density $\mathcal{S}$ is proportional to the hydrostatic strain $\Delta$ (see equation (25)). Assuming that the lattice regions follow linear elasticity, their entropy density becomes

$$
\mathcal{S}=\beta p,
$$

where $p=B \Delta$ is the local hydrostatic stress. We emphasize that this relation does not presume that the lattice is elastically isotropic.

The idea of our method is to apply the elastic continuum expression (41) to the discrete lattice regions surrounding the cluster. Then, the third term in equation (40) can be approximated by the sum

$$
S_{\mathrm{q}}=\beta \sum_{\alpha} p_{\alpha} \Omega_{\alpha}
$$

over all lattice sites $\alpha$ lying outside the cluster. In this sum, $p_{\alpha}$ are local hydrostatic stresses and $\Omega_{\alpha}$ local atomic volumes assigned to individual sites. Although a defini- 
tion of local atomic volume is not unique (Sutton and Vitek 1982, 1983), we assume that these volumes are chosen in such a way that the overall volume of a lattice region equals the sum of individual atomic volumes. Importantly, despite the uncertainty in local atomic volumes, within the embedded-atom method each product $p_{\alpha} \Omega_{\alpha}$ is determined in a unique manner (for example Foiles and Adams (1989)). For a monatomic metal, the quasicontinuum expression (42) can be calculated as

$$
S_{\mathrm{q}}=\frac{\beta}{3} \sum_{\alpha} \sum_{\alpha \neq \gamma}\left[\frac{1}{2} \phi^{\prime}\left(R_{\alpha \gamma}\right)+F^{\prime}\left(\bar{\rho}_{\alpha}\right) \rho^{\prime}\left(R_{\alpha \gamma}\right)\right] R_{\alpha \gamma},
$$

where the second sum runs over all atoms $\gamma$ other than atom $\alpha$. In this expression, $\phi$ and $\rho$ are the pair interaction energy and electron density respectively as functions of the interatomic distance $R_{\alpha \gamma}, F(\bar{\rho})$ is the embedding function and

$$
\bar{\rho}_{\alpha}=\sum_{\gamma \neq \alpha} \rho\left(R_{\alpha \gamma}\right)
$$

is the total electron density on atom $\alpha$. Equation (43) also holds for a pair potential by omitting the embedding-energy term.

Thus, in our method the total point-defect entropy is calculated as the sum

$$
S=\tilde{S}_{N_{*}}+S_{\mathrm{q}}
$$

with $\tilde{S}_{N_{*}}$ and $S_{\mathrm{q}}$ given by equations (10) and (43) respectively. We emphasize that, although this method was inspired by the spherical-model expression (40), the final equation (45) does not require a spherical symmetry of the strain field. Not only the block but also the cluster can have any other shape (e.g. a cubic shape). However, this method does assume that the strained lattice outside the cluster complies with the linear-elasticity approximation and the linear relation between the vibrational entropy and hydrostatic stress. It also assumes that gradients of the hydrostatic stress outside the cluster are small enough to justify the continuum approximation. In contrast with the supercell method, this new method can be readily applied to large simulation blocks because the computation of $S_{\mathrm{q}}$ is a fast linear- $N$ procedure. An advantage over the original embedded-cluster method is that the total defect entropy is obtained from one computational run and does not have to be derived by extrapolation of cluster entropies computed by multiple runs.

The new method was tested by conducting many entropy calculations for a vacancy and an interstitial in copper with various block and cluster sizes, and the results were very encouraging. For example, table 5 summarizes our results for a

Table 5. Vacancy and interstitial formation entropies in copper calculated in the FHA by the elastically corrected embedded-cluster method. The calculations were performed on a 5324-atom cubic supercell with periodic boundary conditions containing a spherical cluster with $N_{*}=459,627$ and 767 atoms. The quasicontinuum entropy $S_{\mathrm{q}}$ (see

\begin{tabular}{|c|c|c|c|c|c|c|c|c|}
\hline \multirow[b]{2}{*}{ Defect } & \multicolumn{4}{|c|}{$S_{p}\left(\right.$ units of $\left.k_{\mathrm{B}}\right)$} & \multicolumn{4}{|c|}{$S_{V}$ (units of $k_{\mathrm{B}}$ ) } \\
\hline & $N_{*}=4.59$ & 627 & 767 & Supercell & $N_{*}=459$ & 627 & 767 & Supercell \\
\hline Vacancy & 1.177 & 1.195 & 1.193 & 1.231 & 2.916 & 2.935 & 2.934 & 2.969 \\
\hline Interstitial & 10.563 & 10.642 & 10.722 & 11.096 & -3.170 & -3.097 & -3.017 & -2.791 \\
\hline
\end{tabular}
equation (43)) was computed with the thermal expansion factor $\beta=5.479 \times 10^{-5}$. The entropies determined by the supercell method are listed for comparison. 
5324-atom cubic supercell with three different sizes of a spherical cluster. If the cluster size is large enough $\left(N_{*}=500-800\right)$, the point-defect entropy almost does not change with $N_{*}$. The obtained entropy values are close to those found in $\S 4.3$ by the supercell method (cf. table 3). A more detailed cluster-size dependence of the defect entropy obtained by this 'elastically corrected' embedded-cluster method is illustrated in figure 5. For $N_{*}>100$, the vacancy entropy follows a linear dependence on $1 / N_{*}$ and extrapolates to $1.212 k_{\mathrm{B}}$, in excellent agreement with the supercell result, $1.231 k_{\mathrm{B}}$. The same agreement was found for an interstitial in copper. The agreement improves still further as we increase the block size.

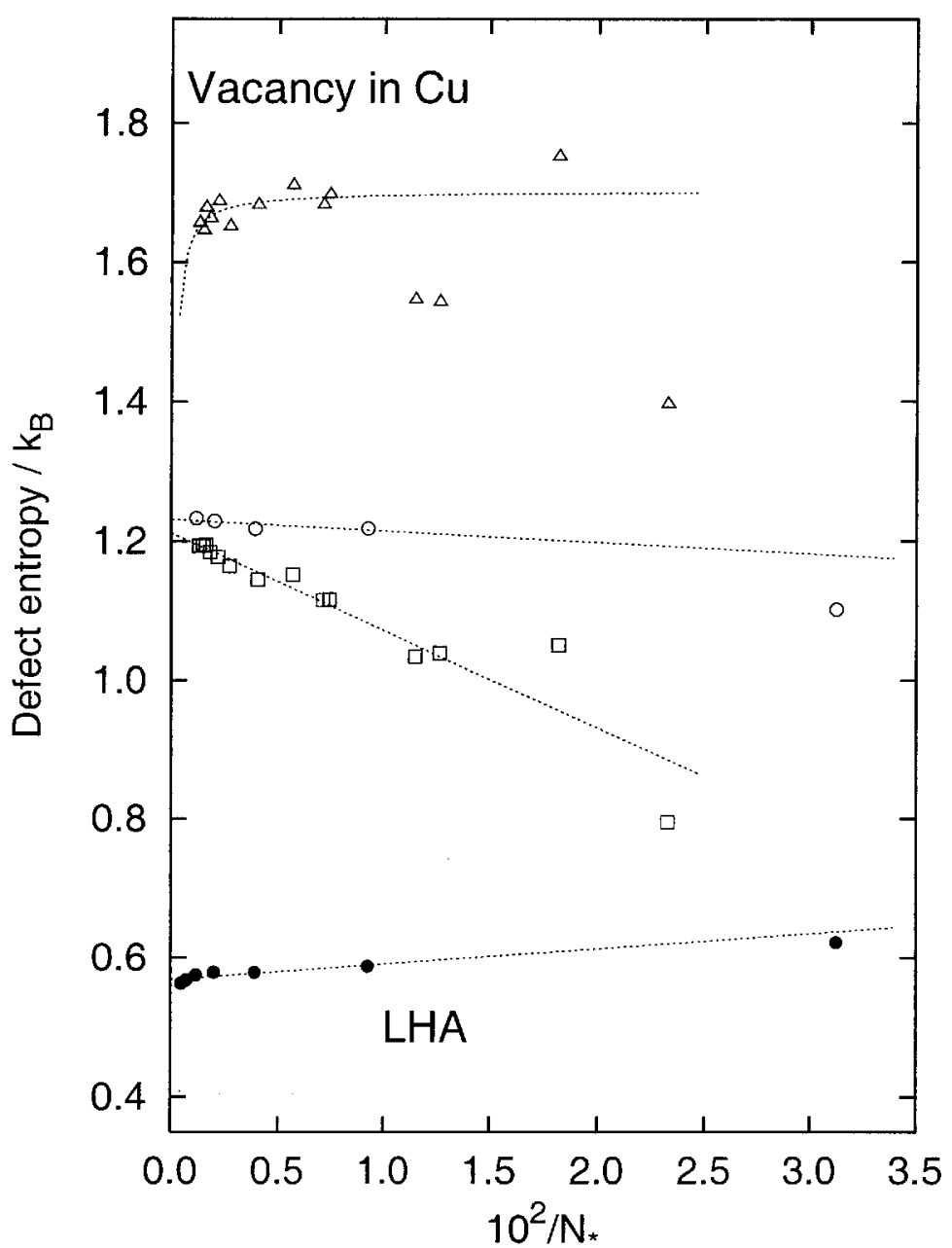

Figure 5. Summary of vacancy formation entropies in copper at $p=0$ as functions of the inverse number $1 / N *$ of dynamic atoms in the simulation block. Calculations were performed in the FHA by the supercell method $(\bigcirc)$, original embedded-cluster method $(\triangle)$ and elastically corrected embedded-cluster method $(\square)$. The embeddedcluster calculations were carried out on a 5324-atom cubic supercell with periodic boundary conditions. The entropies calculated in the LHA (O) are shown for comparison. 


\section{$\S 6$. Discussion AND CONCLUSIONS}

In this paper, problems associated with entropy calculations were analysed for individual point defects in metals. However, those problems are quite general and have significant importance in many other situations. For example, in calculations of jump frequencies of point defects from the harmonic transition state theory (Vineyard 1957), the jump attempt frequency involves the so-called migration entropy of a defect. The latter is calculated by basically the same scheme as the defect formation entropy and is therefore also vulnerable to finite-size effects and boundary conditions. In this case, however, the effect of boundary conditions can be less significant than for defect formation because the number of atoms in the system is conserved and the volume effect is smaller. As another example, the stability of point-defect clusters arising under irradiation or upon quenching depends on their entropy, which in this case includes contributions associated with defect-defect interactions.

In principle, defect formation entropies can be calculated at finite temperatures using molecular dynamics or Monte Carlo methods (De Lorenzi and Jacucci 1986, Foiles 1994). However, because such calculations are complex and computationally expensive, routine calculations are performed by combining molecular statics with the harmonic approximation to atomic vibrations. Within this scheme, there are two different approaches to entropy calculations, called the supercell and the embeddedcluster methods.

The supercell method is the most straightforward and by far the most accurate. It is ideal for a single defect, which can be conveniently simulated with a block containing around $10^{3}$ atoms. In more complex situations that require significantly larger blocks, the application of this method is hampered by numerical problems associated with handling large dynamic matrices. For such situations, the original embeddedcluster method previously offered the only reasonable alternative. Our elasticity analysis shows that the defect entropy determined by the embedded-cluster method equals approximately

$$
S=S_{\mathrm{c}}+\frac{N_{*}}{N} S_{\mathrm{el}},
$$

$S_{\mathrm{c}}$ being the defect core entropy, $S_{\mathrm{el}}$ the elastic part of the entropy and $N_{*}$ the number of atoms in the cluster. This expression includes only a part of the total elastic entropy $S_{\mathrm{el}}$ in proportion to $N_{*} / N$. Therefore, the entropies delivered by the method are essentially incomplete and must be subject to further processing. Namely, the entropies must be computed for a set of different block and/or cluster sizes, and the results must be plotted against $N_{*} / N$. Then, a linear extrapolation to $N_{*} / N \rightarrow 0$ gives the core entropy, while a linear extrapolation to $N_{*} / N \rightarrow 1$ allows us to recover the full entropy $S_{\mathrm{c}}+S_{\mathrm{el}}$. In practice, the accuracy of the method is not high because equation (46) relies on strong approximations, such as spherical symmetry of the elastic field around the defect and the simulation block. Under real conditions, these assumptions are not fulfilled even for a single defect On the other hand, the method provides a very accurate estimate of the core entropy of an individual defect, which is a useful quantity by itself. Unlike the full defect formation entropy, the defect core entropy does not depend sensitively on the external conditions or the system size and characterizes the degree of local atomic distortions in the defect core region. Note that the defect 
core energy cannot be calculated as easily because of the singularity of the elastic strain-energy density at $r \rightarrow 0$.

As an alternative to the supercell and the embedded-cluster methods, we have proposed a new, elastically corrected embedded-cluster method that seems to alleviate effectively the problems inherent in both previous methods. That is, as in the supercell method, the defect entropy is determined from the entropy change of the whole set of free atoms. However, this entropy change is not calculated exactly but is rather split into two parts (equation (45)) that are calculated in different ways. The first part is the entropy change $\tilde{S}_{N_{*}}$ of an $N_{*}$-atom cluster containing the defect. This part can be determined from equation (10) as in the original embedded-cluster method, but it can also be calculated by any other atomistic simulation method beyond the harmonic approximation, for example by the Monte Carlo method (Foiles 1994). The second part, $S_{\mathrm{q}}$, is the entropy change of the lattice regions surrounding the cluster. This part is calculated from equation (42), which is based on a quasicontinuum approximation of the strained-lattice entropy. This calculation assumes that the strained lattice around the cluster follows the linear-elasticity approximation and the linear relation (41) between the entropy and local hydrostatic stress. Since the calculation of $S_{q}$ is a fast linear- $N$ procedure, the computation time of the method is dominated by the calculation of $\tilde{S}_{N_{*}}$. In other words, the entropy associated with the lattice regions around the defect is included at almost no cost. In contrast with the original embedded-cluster method, the total defect entropy is obtained by making just one computational run. The obtained entropy can be further refined by a linear extrapolation to $1 / N_{*} \rightarrow 0$ with a fixed $N$, as illustrated in figure 5. These computational advantages make the elastically corrected embedded-cluster method applicable to much larger simulation blocks, which in turn may allow us to perform entropy calculations for more complex systems, including those involving multiple length scales. For example, the new method can be useful in modelling point defects in or near grain boundaries, dislocations and other extended defects.

Figure 5 summarizes the vacancy formation entropies in copper calculated by different methods as functions of the inverse number of dynamic atoms in the simulation block. This figure illustrates that, for a given number of dynamic atoms $N_{*}>500$ (and thus a given amount of computational efforts), the elastically corrected embedded-cluster method provides an accuracy comparable to that of the supercell method, but can be applied to much larger simulation blocks. The entropies obtained by the original embedded-cluster method show a larger scatter of data points and do not approach the defect formation entropy, at least not within the range of accessible cluster sizes. As was shown by Fernandez et al. (2000) and confirmed in this paper, the entropy predicted by this method diverges (tends to $+\infty$ or $-\infty$ ) when extrapolated to $1 / N_{*} \rightarrow 0$. Figure 5 also demonstrates that the LHA, although very fast, provides a very poor approximation of the defect entropy.

In the elastically corrected embedded-cluster method, the cluster must encompass all atoms around the defect core that experience significant (nonlinear) displacements from their perfect-lattice positions. Indeed, the idea behind the basic relations (25) and (41) assumed in this method is that the entropy density $\mathcal{S}$ of a uniformly strained crystal, being a scalar quantity, can depend only on geometric invariants of the strain tensor. If the strain is small, $\mathcal{S}$ must be proportional to the first-order invariant, that is to the volume deformation $\Delta$ or, assuming linear elasticity, to the hydrostatic stress $p$. For higher strain levels, equation (25) would need 
to be modified by including second- or higher-order invariants, while equation (41) might need to include nonlinear elasticity corrections. Moreover, even for sufficiently small strains, it should be remembered that the quasicontinuum approximation (42) neglects strain gradients, which makes it similar in spirit to the local-density approximation in electronic theory (Kohn and Shan 1965). Our quasicontinuum approximation can, in principle, be improved by including strain-gradient corrections. Such higher-order quasicontinuum approximations involving nonlinear effects and gradient corrections lie beyond the scope of this paper.

\section{ACKNOWLEDGEMENTS}

Y. M. wishes to thank the US Department of Energy (DOE), Office of Basic Energy Sciences (BES), for the financial support of this work (contract DE-FG0299ER45769). A. F. V. is also grateful to DOE, BES, for financial support. The work at Los Alamos National Laboratory was supported by DOE under contract W-7405-ENG-36 (M. R. S. and A. F. V.).

\section{APPENDIX A}

\section{ElaSTIC STRAIN FIELD AROUND A POINT DEFECT}

The elastic displacement field around a point defect located in the centre of a sphere of radius $R$ (figure 1) is given by (Eshelby 1956, Landau and Lifshitz 1986)

$$
u(r)=a r+\frac{b}{r^{2}},
$$

with the boundary condition $u\left(r_{\mathrm{c}}\right)=u_{\mathrm{c}}$. The first term in this equation appears owing to image forces, which also make $a$ and $b$ functions of $R$. These functions depend on the boundary condition at $r=R$.

If the external pressure is zero $(p=0)$, the boundary condition at $r=R$ is zero radial stress:

$$
\sigma_{r r}(R)=0 \text {. }
$$

Using the appropriate expression for $\sigma_{r r}$, we find that

$$
\begin{gathered}
a=\frac{2(1-2 \nu) u_{\mathrm{c}} r_{\mathrm{c}}^{2}}{(1+\nu) R^{3}}\left(1+\frac{2(1-2 \nu) r_{\mathrm{c}}^{3}}{(1+\nu) R^{3}}\right)^{-1}, \\
b=u_{\mathrm{c}} r_{\mathrm{c}}^{2}\left(1+\frac{2(1-2 \nu) r_{\mathrm{c}}^{3}}{(1+\nu) R^{3}}\right)^{-1} .
\end{gathered}
$$

If the volume of the sphere is fixed ( $V=$ constant), the boundary condition is

$$
u(R)=0 \text {, }
$$

and we obtain

$$
a=-\frac{u_{\mathrm{c}} r_{\mathrm{c}}^{2}}{R^{3}}\left(1-\frac{r_{\mathrm{c}}^{3}}{R^{3}}\right)^{-1},
$$




$$
b=u_{\mathrm{c}} r_{\mathrm{c}}^{2}\left(1-\frac{r_{\mathrm{c}}^{3}}{R^{3}}\right)^{-1} .
$$

Considering $\left(r_{\mathrm{c}} / R\right)^{3}$ as a small parameter and limiting our calculations to linear terms in that parameter, the above equations become

$$
\begin{gathered}
a=\frac{2(1-2 \nu) u_{\mathrm{c}} r_{\mathrm{c}}^{2}}{(1+\nu) R^{3}}\left(1-\frac{2(1-2 \nu) r_{\mathrm{c}}^{3}}{(1+\nu) R^{3}}\right) \\
b=u_{\mathrm{c}} r_{\mathrm{c}}^{2}\left(1-\frac{2(1-2 \nu) r_{\mathrm{c}}^{3}}{(1+\nu) R^{3}}\right)
\end{gathered}
$$

for $p=0$, and

$$
\begin{gathered}
a=-\frac{u_{\mathrm{c}} r_{\mathrm{c}}^{2}}{R^{3}}\left(1+\frac{r_{\mathrm{c}}^{3}}{R^{3}}\right), \\
b=u_{\mathrm{c}} r_{\mathrm{c}}^{2}\left(1+\frac{r_{\mathrm{c}}^{3}}{R^{3}}\right),
\end{gathered}
$$

for $V=$ constant. We note that for an infinitely large system $(R \rightarrow \infty)$ these equations give $a=0$ and $b=u_{\mathrm{c}} r_{\mathrm{c}}^{2}$.

\section{REFERENCES}

Catlow, C. R. A., 1997, New Trends in Materials Chemistry, edited by C. R. A. Catlow and A. Cheetham (Dordrecht: Kluwer), p. 141.

Catlow, C. R. A., Corish, J., Jacobs, P. W. M., and Lidiard, A. B., 1981, J. Phys. C, 14, L121.

Debiaggi, S. B., Decorte, P. M., and Monti, A. M., 1996, Phys. Stat. sol. (b) , 195, 37.

De Lorenzi, G., and Jacucci, G., 1986, Phys. Rev. B, 33, 1993.

Eshelby, J. D., 1956, Solid St. Phys., 3, 79.

Fernandez, J. R., and Monti, A. M., 1993, Phys. Stat. sol. (b), 179, 337.

Fernandez, J. R., Monti, A. M., and Pasianot, R. C., 1996, J. nucl. Mater., 229, 1; 2000, Phys. status solidi (b), 219, 245.

Finnis, M. W., and SACHDev, M., 1976, J. Phys. F, 6, 965.

Flynn, C. P., 1972, Point Defects and Diffusion (Oxford: Clarendon).

Folles, S. M., 1994, Phys. Rev. B, 49, 14930.

Foiles, S. M., and Adams, J. B., 1989, Phys. Rev. B, 40, 5909.

Frank, W., Breier, U., Elsässer, C., and Fähnle, M., 1996, Phsy. Rev. Lett., 77, 518.

Gillan, M. J., 1981, Phil. Mag. A, 43, 301.

Harding, J. H., 1990, Rep. Prog. Phys., 53, 1403.

Hatcher, R. D., Zeller, R., and Dederichs, P. H., 1979, Phys. Rev. B, 19, 5083.

Kohn, W., and Sham, L. J., 1965, Phys. Rev., 140, A1133.

Landau, L. D., and Lifshitz, E. M., 1980, Statistical Physics, Part 1, third edition (Oxford: Pergamon); 1986, Theory of Elasticity, third edition (Oxford: Pergamon).

LeSar, R., Najafabadi, R., and Srolovitz, D. J., 1989, Phys. Rev. Lett., 63, 624.

Sutton, A. P., 1989, Phil. Mag. A, 60, 147; 1992, Phil. Trans. R. Soc. A, 341, 233.

Sutton, A. P., and Vitek, V., 1982, Acta metall., 30, 2011; 1983, Phil. Trans. R. Soc. A, 309, 1

Vineyard, G. H., 1957, J. Phys. Chem. Solids, 3, 121.

Voter, A. F., 1993, Unclassified Technical Report LA-UR 93-3901 Los Alamos National Laboratory; 1994, Intermetallic Compounds, Vol. 1 (New York: Wiley), chapter 4, p. 77; 1998, Phys. Rev. B, 57, 13985. 